\title{
Association between the Risk Factors for Pancreatic Ductal Adenocarcinoma and Those for Malignant Intraductal Papillary Mucinous Neoplasm
}

\author{
Ken Kamata ${ }^{a}$ Mamoru Takenaka ${ }^{a}$ Atsushi Nakai ${ }^{a}$ Shunsuke Omoto ${ }^{a}$

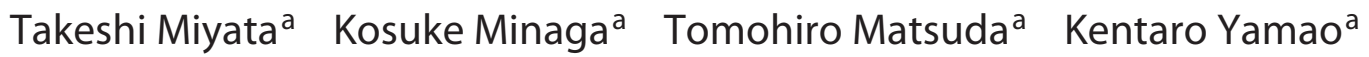 \\ Hajime Imai $^{a}$ Yasutaka Chiba ${ }^{d}$ Toshiharu Sakurai ${ }^{a}$ Tomohiro Watanabe ${ }^{a}$ \\ Naoshi Nishida $^{a}$ Takaaki Chikugo $^{b}$ Ippei Matsumoto ${ }^{c}$ Yoshifumi Takeyamac $^{c}$ \\ Masatoshi Kudo ${ }^{a}$ \\ Departments of a Gastroenterology and Hepatology, b Pathology, and ' Surgery, Kindai University Faculty of \\ Medicine, and ${ }^{\mathrm{d}}$ Clinical Research Center, Kindai University Hospital, Osaka-Sayama, Japan
}

\section{Keywords}

Intraductal papillary mucinous neoplasm · Pancreatic ductal adenocarcinoma $\cdot$ Smoking history $\cdot$ Risk factors

\begin{abstract}
Background and Aims: Risk factors for pancreatic ductal adenocarcinoma (PDAC) include diabetes mellitus, chronic pancreatitis, obesity, a family history of pancreatic cancer, and a history of smoking or alcohol consumption. The aim of this study was to evaluate the association between risk factors for PDAC and malignant intraductal papillary mucinous neoplasm (IPMN). Methods: The study included 134 consecutive patients with IPMN who underwent surgical resection at Kindai University Hospital between April 2009 and March 2015. Data on the presence or absence of mural nodules (MNs) and risk factors for PDAC were evaluated.
\end{abstract}

Multivariable logistic regression analysis was performed with malignant IPMN as the outcome variable and MNs and risk factors for PDAC as explanatory variables. Results: The odds ratio of malignant IPMN to MNs was 3.88 (95\% confidence interval $[\mathrm{Cl}] 1.53-9.84 ; p=0.004)$, whereas that of malignant IPMN to smoking history was 1.66 (95\% Cl 0.74-3.71; $p=0.22$ ). When the presence of MNs was considered as a predictive factor for malignancy, the sensitivity and specificity were 88.5 and $32.1 \%$, respectively, whereas when the presence of both smoking history and MNs was considered, the specificity improved to $73.2 \%$, with a decrease in sensitivity to $42.3 \%$. Conclusions: The presence of both a smoking history and MNs was a valuable predictive factor for malignant IPMN with high specificity. A smoking history should be considered before surgical resection in addition to the presence of MNs.

(c) 2017 S. Karger AG, Basel

\section{KARGER}

(C) 2017 S. Karger AG, Basel

E-Mail karger@karger.com

www.karger.com/ocl
Dr. Mamoru Takenaka

Department of Gastroenterology and Hepatology

Kindai University Faculty of Medicine

377-2 Ohno-Higashi, Osaka-Sayama, Osaka 589-8511 (Japan)

E-Mail mamoxyo45@gmail.com 


\section{Introduction}

Intraductal papillary mucinous neoplasm (IPMN) is diagnosed by several imaging modalities, such as ultrasonography, computed tomography, magnetic resonance cholangiopancreatography, and endoscopic ultrasonography. The 2012 international consensus guidelines for the management of IPMN define the presence of mural nodules (MNs) as high-risk stigmata, and IPMN with $\mathrm{MN}$ is an indication for surgical resection [1]. Pathologically, IPMN is classified as low, intermediate, or highgrade dysplasia or as invasive [2]. Invasive IPMN at an advanced stage mimics ordinary pancreatic ductal adenocarcinoma (PDAC). Diabetes mellitus (DM), chronic pancreatitis (CP), obesity, a family history of pancreatic cancer, a smoking history, and alcohol consumption are well-known risk factors for PDAC [3-8]. However, the association of these risk factors with malignant IPMN, including high-grade dysplasia and invasive disease, is poorly understood. In this study, we evaluated a database of 134 consecutive patients with IPMN who underwent surgical resection and assessed the association of risk factors for PDAC with malignant IPMN.

\section{Patients and Methods}

\section{Study Design}

The present study was a single-center retrospective study using prospectively collected data. The final diagnoses were made according to histological specimens obtained during surgical resection. This study was performed with the approval of the ethics committee of Kinki University School of Medicine.

\section{Patients}

A total of 134 consecutive patients with IPMN who underwent surgical resection for IPMN at our hospital between April 2009 and March 2015 were included. Patients who underwent surgical resection for PDAC concomitant with IPMN were excluded from the study. Data were collected prospectively before surgical resection and included the presence or absence of MNs, DM (HbAlc [National Glycohemoglobin Standardization Program] $\geq 6.5$ ), CP, obesity (body mass index $>30$ ), family history of pancreatic cancer, smoking history (former and current smoker), and alcohol consumption ( $>37.5 \mathrm{~g} /$ day). For DM, information on disease duration and the presence or absence of rapid progression was also collected. For smoking history, pack-years data were also collected.

\section{Definitions}

IPMN was classified into main duct type, mixed type, or branch duct type based on imaging studies and according to the 2012 international consensus guidelines [1]. The reference standard was the pathological findings after surgical resection. Malignant IPMN was defined as high-grade dysplasia or invasive disease. Benign IPMN was defined as low- and moderate-grade dysplasia and bor-

Risk Factors for Malignant Intraductal

Papillary Mucinous Neoplasm
Table 1. Patient characteristics $(n=134)$

$\begin{array}{ll}\text { Mean age } \pm \text { SD, years } & 69 \\ \text { Gender, } n & \\ \quad \text { Male } & 69 \\ \quad \text { Female } & 65 \\ \text { Location of resected IPMN, } n & \\ \quad \text { Head } & 94 \\ \text { Body } & 30 \\ \text { Tail } & 10 \\ \text { Type of IPMN, } n & \\ \quad \text { Main duct type } & 19 \\ \quad \text { Mixed type } & 41 \\ \quad \text { Branch duct type } & 74 \\ \text { Final diagnosis, } n & \\ \quad \text { Malignant IPMN } & 78 \\ \quad \text { Benign IPMN } & \end{array}$

SD, standard deviation; IPMN, intraductal papillary mucinous neoplasm. ${ }^{a}$ High-grade dysplasia and invasive. ${ }^{\mathrm{b}}$ Low- and intermediate-grade dysplasia.

derline malignancy. $\mathrm{MN}$ was defined as a protrusion of the cyst wall into its lumen that was detected by imaging modalities, such as ultrasonography, computed tomography, magnetic resonance cholangiopancreatography, or endoscopic ultrasonography. Former smokers were defined as patients who had stopped smoking $>1$ year before surgical resection. Current smokers were defined as patients who were smoking at the time of surgery.

\section{Statistical Analysis}

To examine factors affecting malignant IPMN, a multivariable logistic regression analysis was performed in which the outcome variable was malignant IPMN and the explanatory variables were MNs, DM, CP, obesity, family history of pancreatic cancer, smoking history, and alcohol consumption. The sensitivity, specificity, and accuracy for diagnosing malignant IPMN using MNs and/or risk factors for PDAC were calculated. All statistical analyses were performed using JMP software version 13 (SAS Institute, Cary, NC, USA).

\section{Results}

The characteristics of the 134 patients are shown in Table 1. According to the pathological classification, the number of malignant and benign IPMNs was 78 (58\%) and $56(42 \%)$, respectively. The presence or absence of MNs and/or the risk factors for PDAC in the 134 patients are shown in Table 2. MNs were preoperatively detected in 107 patients and not detected in 27 patients. Table 3 shows the association between the presence or absence of risk factors and malignant or benign IPMN. All patients with CP had malignant IPMN; CP was excluded from the

Oncology 2017;93(suppl 1):102-106 
Table 2. MNs and risk factors for PDAC $(n=134)$

\begin{tabular}{lr}
\hline MN in cyst, $n$ & 107 \\
Present & 27 \\
Absent & \\
\hline DM (HbA1c [NGSP] $\geq 6.5 \%), n$ & 36 \\
Present & \\
Disease duration & 31 \\
$\quad$ Within 3 years & 5 \\
$\quad$ More than 3 years & 98 \\
Absent & \\
\hline CP, $n$ & 9 \\
Present & 125 \\
Absent & \\
\hline Obesity (BMI $>30), n$ & 9 \\
Present & 125 \\
Absent & \\
\hline Smoking history, $n$ & 58 \\
Present & \\
Pack-years & 32 \\
$\quad$ More than 40 & 25 \\
20-39 & 109 \\
1-19 & 76 \\
Absent & \\
\hline Alcohol consumption $(>37.5$ g/day), $n$ & \\
Present & \\
Absent & \\
\hline
\end{tabular}

Family history of pancreatic cancer, $n$

$\begin{array}{lr}\text { Present } & 7 \\ \text { Absent } & 127\end{array}$

MN, mural nodule; PDAC, pancreatic ductal adenocarcinoma; DM, diabetes mellitus; NGSP, National Glycohemoglobin Standardization Program; CP, chronic pancreatitis; BMI, body mass index.

multivariable logistic regression analysis for malignant IPMN despite the strong association of $\mathrm{CP}$ with malignant IPMN.

Patients with DM within 3 years of disease duration had high rates of malignant IPMN. Regarding smoking history, the number of pack-years was positively related to the rate of malignant IPMN and the presence of MNs.

\section{Multivariable Logistic Regression Analysis Using \\ Malignant IPMN as an Outcome Variable}

The results of the multivariable logistic regression analysis are shown in Table 4. Using a cutoff value of $p=$ 0.25 , the presence of MNs and smoking history were identified as factors positively associated with malignant
Table 3. Association between $\mathrm{MNs}$ and other risk factors and malignant or benign IPMN

\begin{tabular}{|c|c|c|}
\hline & $\begin{array}{l}\text { Benign } \\
(n=56)\end{array}$ & $\begin{array}{l}\text { Malignant } \\
(n=78)\end{array}$ \\
\hline \multicolumn{3}{|l|}{ MNs, $n$} \\
\hline Absent & 18 & 9 \\
\hline Present & 38 & 69 \\
\hline \multicolumn{3}{|l|}{$\mathrm{DM}, n$} \\
\hline Absent & 43 & 55 \\
\hline Present & 13 & 23 \\
\hline \multicolumn{3}{|l|}{ Disease duration } \\
\hline Within 3 years & 1 & 4 \\
\hline More than 3 years & 12 & 19 \\
\hline \multicolumn{3}{|l|}{$\mathrm{CP}, n$} \\
\hline Absent & 56 & 69 \\
\hline Present & 0 & 9 \\
\hline \multicolumn{3}{|l|}{ Obesity, $n$} \\
\hline Absent & 52 & 73 \\
\hline Present & 4 & 5 \\
\hline \multicolumn{3}{|l|}{ Smoking, $n$} \\
\hline Absent & 37 & 39 \\
\hline Present & 19 & 39 \\
\hline \multicolumn{3}{|l|}{ Pack-years } \\
\hline More than 40 & 10 & 22 \\
\hline $20-39$ & 8 & 17 \\
\hline $1-19$ & 1 & 0 \\
\hline \multicolumn{3}{|l|}{ Alcohol, $n$} \\
\hline Absent & 48 & 61 \\
\hline Present & 8 & 17 \\
\hline \multicolumn{3}{|l|}{ Family history, $n$} \\
\hline Absent & 51 & 76 \\
\hline Present & 5 & 2 \\
\hline
\end{tabular}

$\mathrm{MN}$, mural nodule; IPMN, intraductal papillary mucinous neoplasm; DM, diabetes mellitus; CP, chronic pancreatitis.

IPMN. Family history was negatively associated with malignant IPMN and was excluded from further analysis.

\section{Sensitivity, Specificity, and Accuracy for Diagnosing} Malignant IPMN Using MNs and/or Smoking History

As an additional analysis, we assessed the accuracy of MNs and/or smoking history for the diagnosis of malignant IPMN considering the results of the multivariable logistic regression analysis. When the presence of MNs was considered an indicator of malignancy, the sensitivity, specificity, positive predictive value (PPV), negative predictive value, and accuracy with $95 \%$ confidence inter- 
Table 4. Multivariable logistic regression analysis of the association between $\mathrm{MNs}$ and the risk factors for PDAC and malignant IPMN ${ }^{\mathrm{a}}$

\begin{tabular}{|c|c|c|c|}
\hline & $\begin{array}{l}\text { Odds } \\
\text { ratio }\end{array}$ & $95 \% \mathrm{CI}$ & $\begin{array}{l}p \\
\text { value }\end{array}$ \\
\hline \multicolumn{4}{|l|}{ Malignant IPMN (outcome variable) } \\
\hline $\mathrm{MN}$ & 3.88 & $1.53-9.84$ & 0.004 \\
\hline $\mathrm{DM}$ & 1.41 & $0.58-3.43$ & 0.45 \\
\hline Obesity & 0.52 & $0.12-2.24$ & 0.38 \\
\hline Smoking history & 1.66 & $0.74-3.71$ & 0.22 \\
\hline Alcohol consumption & 1.40 & $0.48-4.05$ & 0.54 \\
\hline Family history of pancreatic cancer & 0.22 & $0.04-1.34$ & 0.10 \\
\hline
\end{tabular}

$\mathrm{MN}$, mural nodule; PDAC, pancreatic ductal adenocarcinoma; IPMN, intraductal papillary mucinous neoplasm; CI, confidence interval; DM, diabetes mellitus. ${ }^{\text {a }} \mathrm{CP}$ was excluded from this analysis because the initial analysis showed that the value of $\mathrm{CP}$ was unstable.

vals were $88.5 \%$ (82.7-93.2), 32.1\% (24.1-38.8), $64.5 \%$ (60.3-68.0), 66.7\% (49.9-80.4), and 64.9\% (58.2-70.5), respectively. The specificity of the diagnosis using only the presence of MNs was low; therefore, we evaluated the diagnostic ability using both MNs and smoking history. Considering both the presence of MNs and a positive smoking history as indicators of malignancy, the sensitivity, specificity, PPV, negative predictive value, and accuracy with $95 \%$ confidence intervals were $42.3 \%$ (35.4$48.4), 73.2 \%$ (63.6-81.7), 68.8\% (57.6-78.6), 47.7\% (41.4$53.2)$, and $55.2 \%(47.2-62.3)$, respectively.

\section{Discussion}

The frequency of high-grade dysplasia or invasive main duct type IPMN is approximately $70 \%[1,9]$. By contrast, the rate of high-grade dysplasia or invasive branch duct type IPMN is lower; therefore, the indications for the surgical resection of IPMNs, especially for branch duct IPMNs, remain controversial $[1,9]$. In this study, we evaluated 134 patients with resected IPMNs and found that $58.2 \%$ were malignant. MNs are considered as high-risk stigmata, and 107 of the 134 patients (79.9\%) underwent surgical resection because of the presence of MNs; however, the PPV of MNs for malignant IPMN was $64.5 \%$, which was not a satisfactory result. The identification of factors with good predictive value for malignant IPMN is necessary.

Several studies investigated the association of environmental, personal, and hereditary risk factors with the oc-

Risk Factors for Malignant Intraductal

Papillary Mucinous Neoplasm currence or degree of IPMN [10-14]. Capurso et al. [10] compared 390 patients with IPMN with 390 matched controls and found that a history of DM, CP, and a family history of pancreatic cancer were all independent risk factors for IPMN. Carr et al. [11] examined the association between IPMN malignant progression and smoking in 324 resected IPMNs and found no significant difference in the percentage of invasive IPMNs between smokers and nonsmokers ( 22 vs. $18 \%, p=0.5$ ). However, among patients with invasive IPMN, smokers were younger than nonsmokers at the time of diagnosis (65 vs. 72 years, $p=0.01$ ). This indicated that smoking might promote IPMN malignant progression. Rezaee et al. [12] reported that patients with main duct type IPMNs were more frequently smokers than patients with mixed and branch duct type IPMNs $(p=0.03)$. However, there was no difference between the 2 groups in terms of the risk of high-grade dysplasia or invasive carcinoma.

The present study showed no significant difference in the incidence of malignant IPMNs between smoking and nonsmoking groups. However, the odds ratio of smoking (1.66) in patients with malignant IPMN was higher than that of environmental, personal, and hereditary risk factors. Moreover, considering a positive smoking history in combination with the presence of MNs as indications for surgery, the PPV and specificity for malignant IPMN increased from 64.5 to $68.8 \%$ and from 32.1 to $73.2 \%$, respectively, although the accuracy decreased.

This study had several limitations. The study used a retrospective design and included a small number of patients who underwent surgical resection. The results of the analysis should be validated in a prospective larger study. CP was excluded from the multivariable logistic regression analysis of the association between MNs and the risk factors for PDAC and malignant IPMN because the value of $\mathrm{CP}$ was unstable, as all patients with $\mathrm{CP}$ had malignant IPMNs. However, CP may be associated with malignant IPMN.

In conclusion, there was no significant association between the risk factors for PDAC and malignant IPMN. The presence of several risk factors, especially a positive smoking history in addition to the presence of MNs, is an indication for surgical resection in patients with IPMN.

\section{Disclosure Statement}

There are no potential competing interests concerning this study.

Oncology 2017;93(suppl 1):102-106

DOI: $10.1159 / 000481234$ 


\section{Author Contributions}

Ken Kamata: writing of the manuscript, study conception and design, and performing endoscopic ultrasonography. Mamoru Takenaka: writing of the manuscript, drafting conception and design. Atsushi Nakai: data collection. Shunsuke Omoto: data collection. Takeshi Miyata: data collection. Kosuke Minaga: data collection. Tomohiro Matsuda: data collection. Kentaro Yamao: data collection. Hajime Imai: data collection. Yasutaka Chiba: statistical analysis. Toshiharu Sakurai, Tomohiro Watanabe, and Naoshi Nishida: contribution to writing and revising the manuscript. Takaaki Chikugo: pathological evaluation. Ippei Matsumoto and Yoshifumi Takeyama: surgical resection. Masatoshi Kudo: writing of the manuscript and drafting conception and design. Guarantor of the manuscript: Mamoru Takenaka.

\section{References}

1 Tanaka M, Fernández-del Castillo C, Adsay $\mathrm{V}$, et al: International consensus guidelines 2012 for the management of IPMN and MCN of the pancreas. Pancreatology 2012;12:183197.

2 Adsay NV, FukushimaN, Furukawa T, et al: Intraductal neoplasms of the pancreas; in Bosman FT, Hruban RH, Cameiro F, et al (eds): WHO Classification of Tumors of the Digestive System. WHO Classification of Tumors, ed 4. Lyon, IARC, 2010, pp 304-313.

3 Ben Q, Xu M, Ning X, et al: Diabetes mellitus and risk of pancreatic cancer: a meta-analysis of cohort studies. Eur J Cancer 2011;47:19281937.

4 Ueda J, Tanaka M, Ohtsuka T, et al: Surgery for chronic pancreatitis decreases the risk for pancreatic cancer: a multicenter retrospective analysis. Surgery 2013;153:357-364.

5 Lin Y, Kikuchi S, Tamakoshi A, et al: Obesity, physical activity and the risk of pancreatic cancer in a large Japanese cohort. Int J Cancer 2007;120:2665-2671.
6 Jacobs EJ, Chanock SJ, Fuchs CS, et al: Family history of cancer and risk of pancreatic cancer: a pooled analysis from the Pancreatic Cancer Cohort Consortium (PanScan). Int J Cancer 2010;127:1421-1428.

7 Matsuo K, Ito H, Wakai K, et al: Cigarette smoking and pancreas cancer risk: an evaluation based on a systematic review of epidemiologic evidence in the Japanese population. Jpn J Clin Oncol 2011;41:1292-1302.

8 Tramacere I, Scottie L, Jenab M, et al: Alcohol drinking and pancreatic cancer risk: a metaanalysis of the dose-risk relation. Int J Cancer 2010;126:1474-1486.

9 Tanaka M, Chari S, Adsay V, et al: International consensus guidelines for management of intraductal papillary mucinous neoplasms and mucinous cystic neoplasms of the pancreas. Pancreatology 2006;6:17-32.
10 Capurso G, Boccia S, Salvia R, et al: Risk factors for intraductal papillary mucinous neoplasm (IPMN) of the pancreas: a multicentre case-control study. Am J Gastroenterol 2013; 108:1003-1009.

11 Carr RA, Roch AM, Shaffer K, et al: Smoking and IPMN malignant progression. Am J Surg 2017;213:494-497.

12 Rezaee N, Khalifian S, Cameron JL, et al: Smoking is not associated with severe dysplasia or invasive carcinoma in resected intraductal papillary mucinous neoplasms. J Gastrointest Surg 2015;19:656-665.

13 Leal JN, Kingham TP, D'Angelica MI, et al: Intraductal papillary mucinous neoplasms and the risk of diabetes mellitus in patients undergoing resection versus observation. J Gastrointest Surg 2015;19:1974-1981.

14 Sturm EC, Roch AM, Shaffer KM, et al: Obesity increases malignant risk in patients with branch-duct intraductal papillary mucinous neoplasm. Surgery 2013;154:803-809. 